Planetary Systems in the Universe - Observation, Formation and Evolution

Proceedings IAU Symposium No. 202, (C)2004 IAU

Alan Penny, Pawel Artymowicz, Anne-Marie Lagrange, 8 Sara Russell, eds.

\title{
ELODIE low-mass companions to solar-type stars
}

\author{
D. Naef, M. Mayor, D. Queloz, S. Udry \\ Geneva Observatory, CH-1290 Sauverny, Switzerland
}

F. Arenou

DASGAL, Observatoire de Paris, F-92195 Meudon CEDEX, France

J.-L. Beuzit, C. Perrier-Bellet

Observatoire de Grenoble, UJF, BP 53, F-38041 Grenoble, France

\author{
J.-P. Sivan
}

Observatoire de Haute-Provence, F-04870 St-Michel, France

\begin{abstract}
We present radial-velocity measurements for three solar-type stars (HD 127506, HD 174457 and HD 185414) hosting low-mass companions. The measurements were obtained with the ELODIE echelle spectrograph mounted on the 1.93-m telescope at Observatoire de Haute-Provence (CNRS, France) within the frame of the OHP-ELODIE Planet Search Programme. The inferred minimum masses of the detected companions are in the substellar mass range. Combining ELODIE radial-velocity data and HIPPARCOS astrometric data, the inclination angles of the orbital planes of HD 127506 and HD 174457 have been derived providing us with the de-projected masses of the companions: $m_{2}=44 \mathrm{M}_{\mathrm{Jup}}$ for the companion of HD 127506 and $m_{2}=0.13 \mathrm{M}_{\odot}$ for the companion of HD 174457. Moreover, using adaptive optics measurements, we show that HD 174457 is probably a $(\mathrm{F} 8 \mathrm{~V}+\mathrm{M} 7 \mathrm{~V}+\mathrm{M} 3-4 \mathrm{~V})$ triple system. To date, only a minimal orbital solution is available for HD 185414 .
\end{abstract}

\section{Introduction}

6 years after the OHP-ELODIE Planet Search Programme (Mayor \& Queloz 1996) was initiated, the number of planetary companions detected with the ELODIE spectrograph (Baranne et al. 1996) reaches 5 (see Sivan et al., this volume). Some stars in our sample exhibit larger radial-velocity variations induced by more massive companions (see e.g. HD 110833 with $m_{2} \sin i=19 \mathrm{M}_{\text {Jup }}$, Mayor et al. 1997). In some cases, it is possible to estimate the inclination of the orbital planes by combining radial-velocity data with astrometric and/or adaptive optics measurements and therefore derive the de-projected masses. Obtaining real masses is mandatory to study the transition between planets and brown dwarfs. 


\section{Orbital solutions and discussion}

Table 1 shows the best orbital solutions fitted to the radial-velocity measurements for HD 127506, HD 174457 and HD 185414. Our radial-velocity measurements are displayed in Fig. 1. The orbital solutions for HD 127506 and HD 185414 are only minimal solutions since we have not covered a full orbital cycle yet. For HD 127506, the 24 radial-velocity measurements obtained with CORAVEL (Baranne et al. 1979) over the last 5000 days are compatible with our fitted period. For HD 185414, the CORAVEL measurements do not give a reliable constraint on the orbital period but they are compatible with the solution of Table 1.

Combining ELODIE radial velocities and HIPPARCOS astrometric data (ESA 1997), the inclination angle of the orbital planes of the companions have been measured for HD 127506 (Halbwachs et al. 2000) and HD 174457 (this contribution). With these angles, the real masses of the companions can be computed (see Table 1).

For HD 174457, we have detected a more distant companion using the PUEO adaptive optics system mounted on the $\mathrm{CFH}$-telescope. The distance between HD 174457 and its visual companion, about $22 \mathrm{AU}$, is clearly not compatible with the separation inferred for the companion inducing the radial-velocity variation. HD 174457 is thus a triple system. The visual companion is $2.53 \mathrm{mag}$ fainter than its primary star in the $\mathrm{K}$-band. Assuming a typical value $M_{\mathrm{K}} \simeq 3.45$ for HD 174457, we infer $M_{\mathrm{K}} \simeq 6$ for the visual companion so this object is probably a M3-M4 dwarf. We have also detected a visual companion to HD 185414 with PUEO but the data reduction is not completed yet so we will not say more about it here.

Table 1. Results of the best Keplerian fit to our radial-velocity measurements

\begin{tabular}{llccc}
\multicolumn{2}{c}{ Parameter } & HD 127506 & HD 174457 & HD 185414 \\
\hline$P$ & days & $2574 \pm 95$ & $845.3 \pm 2.2$ & $1390 \pm 42$ \\
$e$ & & $0.72 \pm 0.03$ & $0.23 \pm 0.01$ & $0.421 \pm 0.009$ \\
$\gamma$ & $\mathrm{km} \mathrm{s}^{-1}$ & $-19.066 \pm 0.031$ & $-26.937 \pm 0.010$ & $-17.656 \pm 0.022$ \\
$K_{1}$ & $\mathrm{~km} \mathrm{~s}^{-1}$ & $0.908 \pm 0.019$ & $1.282 \pm 0.020$ & $1.083 \pm 0.013$ \\
$f_{1}(m)$ & $10^{-5} \mathrm{M}_{\odot}$ & $6.6 \pm 0.9$ & $17.1 \pm 0.8$ & $13.7 \pm 0.7$ \\
$a_{1} \sin i$ & $10^{-3} \mathrm{AU}$ & $148 \pm 9$ & $96.8 \pm 1.6$ & $125.1 \pm 4.1$ \\
$N$ & & 39 & 50 & 26 \\
$\sigma(O-C) \mathrm{m} \mathrm{s}^{-1}$ & 13.2 & 28.1 & 21.1 \\
$m_{2} \sin i$ & $\mathrm{M}_{\text {Jup }}$ & 33.4 & 61.9 & 55.7 \\
$a$ & $\mathrm{AU}$ & 3.26 & 1.84 & 2.52 \\
$m_{2}$ & $\mathrm{M}_{\text {Jup }}$ & $\simeq 44$ & $\simeq 130$ & - \\
\hline \hline
\end{tabular}

\section{References}

Baranne, A., Mayor, M., \& Poncet, J.L. 1979, Vistas Astr., 23, 279

Baranne, A., Queloz, D., Mayor, M., et al., 1996, A\&AS 119, 1

ESA, 1997, The HIPPARCOS and TYCHO catalogue, ESA-SP 1200

Halbwachs, J.L., Arenou, F., Mayor, M., Udry, S., \& Queloz, D. 2000, A\&A, 355, 581 

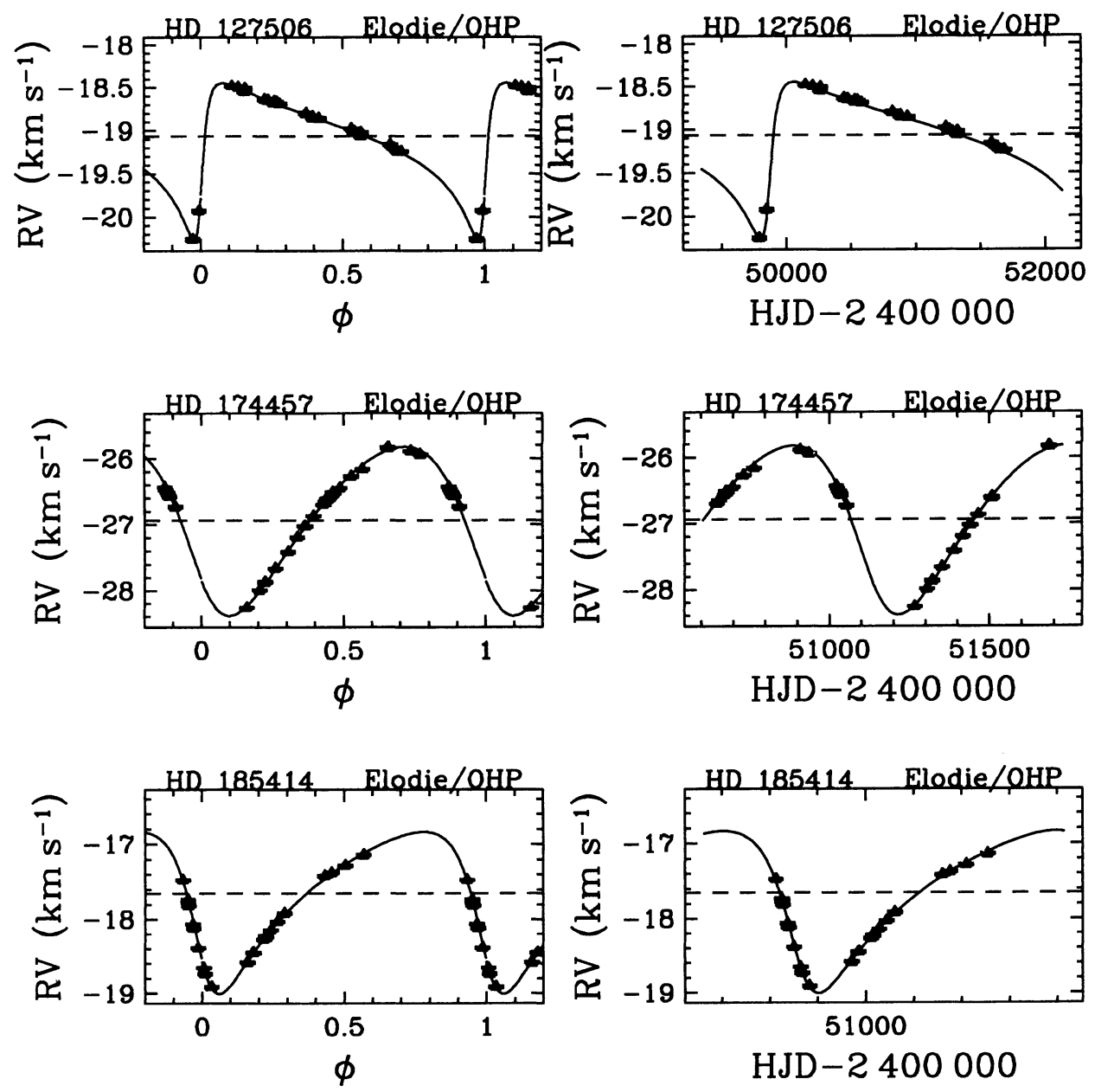

Figure 1. Phase-folded curves and temporal radial velocities for HD 127506, HD 174457 and HD 185414

2000, ApJ, 529, L41

Mayor, M., \& Queloz, D. 1996, in the 9th Cambridge Workshop on Cool Stars, Stellar Systems and the Sun, ASP Conf. Ser., 109

Mayor, M., Queloz, D., Udry, S., \& Halbwachs, J.L. 1997, IAU Coll. 161, 313 